BMJ Paediatrics Open

\section{Assessing the feasibility and acceptability of online measurements of exhaled volatile organic compounds (VOCs) in children with preschool wheeze: a pilot study}

To cite: Holden K, Makinde M, Wilde $\mathrm{M}$, et al. Assessing the feasibility and acceptability of online measurements of exhaled volatile organic compounds (VOCs) in children with preschool wheeze: a pilot study. BMJ Paediatrics Open 2021;5:e001003. doi:10.1136/ bmjpo-2020-001003

$\mathrm{KH}$ and MM are joint first authors.

PM and EAG are joint senior authors.

Received 29 March 2021 Accepted 17 July 2021

Check for updates

(C) Author(s) (or their employer(s)) 2021. Re-use permitted under CC BY-NC. No commercial re-use. See rights and permissions. Published by BMJ.

${ }^{1}$ Leicester NIHR Biomedical Research Centre (Respiratory Theme), Glenfield Hospital, Leicester, UK

${ }^{2}$ Department of Respiratory Sciences, College of Life Sciences, University of Leicester Leicester, UK

${ }^{3}$ Department of Chemistry, University of Leicester, Leicester, UK

${ }^{4}$ Department of Cardiovascular Sciences, University of Leicester Leicester, UK

Correspondence to Dr Erol A Gaillard; eag15@ leicester.ac.uk

\section{ABSTRACT}

Background Investigating airway inflammation and pathology in wheezy preschool children is both technically and ethically challenging. Identifying and validating noninvasive tests would be a huge clinical advance. Real-time analysis of exhaled volatile organic compounds (VOCs) in adults is established, however, the feasibility of this noninvasive method in young children remains undetermined. Aim To determine the feasibility and acceptability of obtaining breath samples from preschool children by means of real-time mass spectrometry analysis of exhaled VOCs.

Methods Breath samples from preschool children were collected and analysed in real time by proton transfer reaction-time of flight-mass spectrometry (PTR-TOF-MS) capturing unique breath profiles. Acetone (mass channel $\mathrm{m} / \mathrm{z} 59$ ) was used as a reference profile to investigate the breath cycle in more detail. Dynamic time warping (DTW) was used to compare VOC profiles from adult breath to those we obtained in preschool children.

Results 16 children were recruited in the study, of which eight had acute doctor-diagnosed wheeze (mean (range) age $3.2(1.9-4.5)$ years) and eight had no history of wheezing (age 3.3 (2.2-4.1) years). Fully analysable samples were obtained in $11(68 \%)$. DTW was used to ascertain the distance between the time series of mass channel m/z 59 (acetone) and the other 193 channels. Commonality of 12 channels $(15,31,33,41,43,51,53$, $55,57,60,63$ and 77 ) was established between adult and preschool child samples despite differences in the breathing patterns.

Conclusion Real-time measurement of exhaled VOCs by means of PTR-MS is feasible and acceptable in preschool children. Commonality in VOC profiles was found between adult and preschool children.

\section{INTRODUCTION}

Wheezing in the preschool years is extremely common. ${ }^{1}$ This group of children represent a diagnostic challenge because they cannot easily perform reliable lung function testing. Furthermore, invasive investigations such as bronchoscopy and obtaining samples by

\section{What is known about the subject?}

Exhaled volatile organic compound (VOC) profiling has been performed in children and preschool children using offline methods and online nanosenso technology.

- Exhaled VOC profiling has been shown to be able to discriminate between those who have respiratory disease and those who do not.

\section{What this study adds?}

It is feasible to collect breath samples from preschool children with wheeze and instantly analyse them by proton transfer reaction-time of flightmass spectrometry for exhaled VOCs in real time.

- There is commonality in the VOC profiles produced in preschool children and adults despite the clear difference in breathing patterns.

bronchoalveolar lavage are not warranted or justifiable in the vast majority of cases. A reliable biomarker associated with steroidresponsive disease, such as sputum or blood eosinophils in adults, has yet to be identified in children. ${ }^{23}$ As such, there is a clear mandate for the identification and validation of non-invasive means of assessing airway inflammation and pathology in these young children.

The area of breath metabolomics, often referred to as 'breathomics' is a rapidly developing technology used to assess respiratory conditions, with promising results in patients with lung cancer, ${ }^{4}$ cystic fibrosis ${ }^{5}$ and asthma. ${ }^{6}$ Metabolites are either released into the blood stream following metabolic processes in tissue or generated directly in the lung such as nitric oxide resulting from 
airway inflammation. ${ }^{7}$ Hundreds of these metabolites exchange across the alveolar membrane and can be detected in human breath. ${ }^{8}$ Offline methodologies such as gas chromatography and two-dimensional gas chromatography combined with mass spectrometry (GC-MS and GC $\times$ GCMS) can provide good separation of the many volatile organic compounds (VOCs) observed in breath. ${ }^{9}$ However, the main disadvantage is that breath is typically collected in a Tedlar bag ${ }^{10}$ or canister ${ }^{11}$ followed by preconcentration of the sample onto a thermal desorption tube or solid phase microextraction fibre. Analysis of a sample after collection often involves transportation, requiring stable storage conditions, extending the total analysis time. Alternatively, online or real-time methods such as proton transfer reaction-MS (PTR-MS) ${ }^{12}$ involve the chemical ionisation (or protonation) of VOCs, which are detected by a time-of-flight (TOF) mass spectrometer providing continuous mass spectral measurements with parts per trillion per volume sensitivity. A unique advantage of real-time VOC monitoring throughout the breath cycle is that it allows for the detection and differentiation of compounds exhaled across the whole of expiration, as opposed to pre-concentration of the combined total or specific phase of the breath cycle. ${ }^{13}$

Profiling exhaled breath VOCs using offline methods has been performed previously in children with asthma ${ }^{14}$ and in preschool children with wheeze. ${ }^{15}$ Few studies using real-time breath analysis have been reported in children ${ }^{16}$ with one study using real-time nanosensor technology in preschool children finding that VOC profiles were different in children with acute wheeze when compared with controls which persisted after recovery from the acute episode. ${ }^{17}$ Real-time collection and analysis of breath VOC profiles has been performed in children aged $4-18$ years with type 1 diabetes mellitus ${ }^{18}$ and chronic kidney disease. ${ }^{19}$ However, we are not aware of reports of real-time collection and analysis of breath VOCs using MS in cohorts of preschool children or preschool children with wheeze.

The principal aims of this pilot feasibility study were to investigate whether it is possible to obtain analysable PTR-MS data, a real-time MS technology, in preschool children and to compare these to samples obtained from adults.

\section{METHODS}

\section{Study population}

Children were recruited from the Leicester Royal Infirmary, UK, aged between 2 and 6 years inclusive with acute wheezing symptoms and controls of the same age range with no wheezing symptoms. Diagnosis of acute wheeze and the decision to be deemed clinically safe to participate was made by the treating physician. For comparison, samples of breath taken from adult patients attending the emergency department at the Leicester Royal Infirmary, UK, were included.

\section{Study protocol}

\section{Selection phase}

The selection phase for the preschool breath samples was conducted over a 10-month period (predominately June and July 2015). Eligible children and their families were approached by a research physician and provided with patient information sheets and given 1 hour to review the information before consenting to participate. A random selection of the breath samples collected from adults attending the emergency department at Leicester Royal Infirmary over a 2-month period was used for comparison.

\section{Sample collection phase}

A 10-min acclimatisation period was given in the sample collection room, which was a quiet and calm setting to enable the participant to become familiar with the breath sampler and the face mask/apparatus. After this period, a background sample was taken for $80 \mathrm{~s}$.

The children were fitted with a disposable, size-appropriate facemask (Intersurgical, Wokingham, Berkshire, UK) attached to an electrostatic bacterial/viral filter (Flexicare Medical Limited, Mountain Ash, Mid Glamorgan, UK) which was connected to the Loccioni breath sampling apparatus (Loccioni Human Care, Sofia, Incentive and Tidal Breath Samplers, Italy). A diagram of the instrument set-up is shown in figure 1. Children performed tidal breathing through the facemask connected to a non-rebreathing valve system, while games, books and toys were provided for them to be distracted with. Measurement of the exhaled VOCs was taken for 80 s with repeat samples being taken using the same process as above after $15 \mathrm{~min}$. The PTR-MS instrumentation was used as described previously in detail by Blake $e t a l^{20}$ The sample line between the apparatus and the instrument was heated to $40^{\circ} \mathrm{C}$ to avoid condensation, reducing loss of VOCs and a heated blanket (Infroheat Limited, Wolverhampton, UK) surrounded the entire sampling apparatus, which was also heated to $40^{\circ} \mathrm{C}$. The heated transfer line was connected to the PTR-TOF-MS. The TOF-MS monitored all of the mass channels $(\mathrm{m} / \mathrm{z})$ simultaneously, providing real-time VOC profiles across a mass range of $\mathrm{m} / \mathrm{z}$ 15-230. Each m/z is the mass of an ion $(\mathrm{m})$ relative to its charge $(\mathrm{z})$.

\section{Breath profile and data analysis}

To ensure any variations observed in the exhaled VOCs between individuals were not an artefact of variations in the background or variations in the abundance of the primary reagent ion $\left(\mathrm{H}_{3} \mathrm{O}^{+}\right)$, the data were normalised as described by Wyche et $a t^{21^{3}}$ (using the same $\mathrm{m} / \mathrm{z}$ ), which is an accepted practice within the field of PTR-MS, and the background signal subtracted.

Acetone $(\mathrm{m} / \mathrm{z} 59)$ was chosen as a reference signal for comparison of VOCs which related to the breath cycle owing to its abundant signal in breath, typically $>100 \mathrm{ppbV}^{22} 23$ While investigating concentrations and distribution of volatile metabolites in the exhaled breath of healthy adult volunteers, Spanel et alidentified acetone as one of the compounds found in higher concentrations with median values of 363 


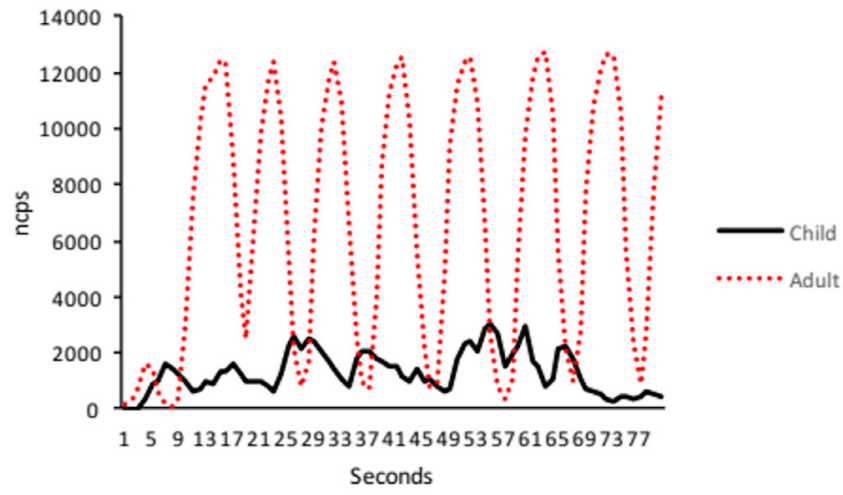

Figure 3 Example of channel 59 (acetone) for a preschool child and an adult. These figures show that when using acetone (ie, an abundant VOC in exhaled breath) as a marker, the adult trace has a regular pattern that represents tidal breathing. In the preschoolers, it is clear that the pattern is irregular, however, the trace for acetone has a similar pattern which corresponds to the capnography trace demonstrating that this methods is measuring exhaled breath VOCs in real time despite the irregular pattern of tidal breathing. VOC, volatile organic compound.

ppbV in exhaled breath. ${ }^{22}{ }^{23}$ Acetone thus appears to be a good candidate to monitor the breath cycle, as a potentially suitable control compound to identify other compounds/ mass channels that specifically vary with the respiratory cycle. Each breath sample was screened for adequacy by assessing the time profile of $\mathrm{m} / \mathrm{z} 59$. Poor samples with no recognisable trace for the $\mathrm{m} / \mathrm{z} 59$ spectrum matching the capnography profile (recorded by the sampler) were discarded at this point without further analysis.

Dynamic time warping (DTW) was used to obtain the distance between the time series of mass channel m/z 59 (acetone) and the remaining mass channels. The DTW library in $\mathrm{R}$ was used to perform $\mathrm{DTW}^{24}$ The underlying principle behind DTW is to compare two time series; a test series $X$ and a reference series $Y$, with the dissimilarity (the DTW distance) between the two series being the extent to which the two series must be deformed or warped in order that the two series resemble each other. The DTW distance provides a very useful measure of similarity between time series and has advantages over the Euclidean distance in that it can accommodate situations where the time axis is not aligned. We used DTW as a measure of similarity between children and adult breath samples as this allows comparison of VOC profiles despite differences in level and pattern of detection.

\section{Patient and public involvement}

This specific study was undertaken with no specific patient (parent) or public involvement (PPI) in the study design, data analysis or distribution of results. However, within our department there has been PPI in the design of other breath collection studies. Furthermore, it is a clear research priority and in the interest of the public that research into VOC analysis as a diagnostic tool is undertaken as per the European Asthma Research Innovation Partnership. ${ }^{25}$

\section{RESULTS}

\section{Participants}

During the study period 54 families were screened of whom 16 met the inclusion criteria and provided written, informed consent to participate in this study. Table 1 displays the demographic and clinical characteristics of the eight preschool children with wheeze and eight controls recruited. Diagnosis of control patients included foreign body in the nose $(n=2)$, fever of unknown origin $(n=1)$, musculoskeletal injury $(n=2)$, swallowed foreign body $(n=1)$, gastrostomy $(n=1)$ and pneumonia $(n=1)$. Eleven of the 16 children recruited $(68 \%, 95 \%$ CI 0.44 to 0.86 ) were able to provide fully analysable breath samples, seven within the acute preschool wheeze group and four within the control group. Five children did not produce a fully analysable sample because they did not keep the facemask on for long enough to allow collection of sufficient breath data. There was no difference between group (preschool wheeze vs control), age or other demographics in ability to produce an analysable sample. None of the children were distressed by the procedure and the procedure was deemed safe by the

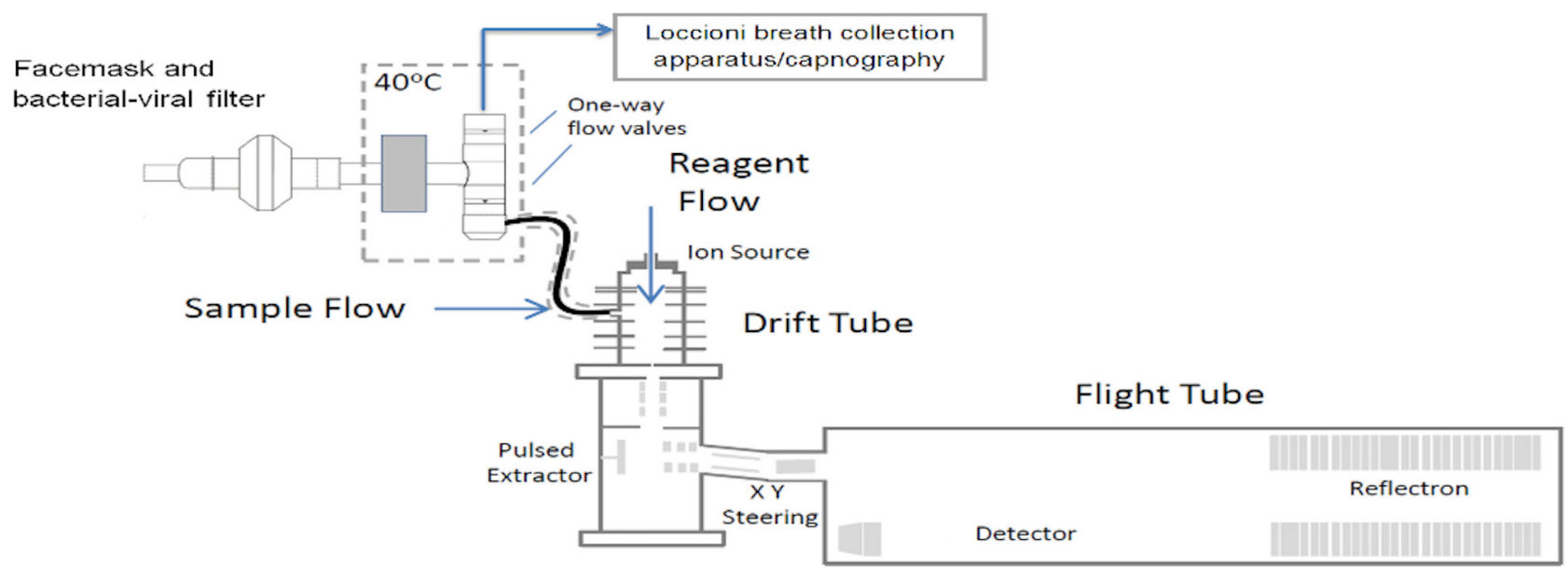

Figure 1 Illustration of apparatus setup. 
Table 1 Clinical characteristics of preschool children

\begin{tabular}{lll} 
& Acute preschool wheeze & Control \\
\hline Participants $(\mathrm{n})$ & 8 & 8 \\
\hline Mean (range) age (years) & $3.2(1.9-4.5)$ & $3.3(2.2-4.1)$ \\
Male, $\mathrm{n}(\%)$ & $4(50)$ & $6(75)$ \\
\hline History of atopy, ${ }^{\mathrm{n}} \mathrm{n}(\%)$ & $6(75)$ & $0(0)$ \\
Use of inhaled corticosteroids (ICS), $\mathrm{n}(\%)$ & $2(25)$ & $0(0)$ \\
Use of short-acting $\beta_{2}$-agonists, $\mathrm{n}(\%)$ & $7(87.5)$ & $0(0)$ \\
\hline History of parental asthma or atopy, ${ }^{*} \mathrm{n}(\%)$ & $6(75)$ & $5(62.5)$ \\
\hline History of parental smoking, $\mathrm{n}(\%)$ & $4(50)$ & $3(37.5)$ \\
\hline History of pets in the family home, $\mathrm{n}(\%)$ & $6(75)$ & $5(62.5)$ \\
\hline
\end{tabular}

*Allergic dermatitis (eczema) or seasonal rhinitis (hay fever).

medical and the research team with no adverse incidents reported $(0 \%, 95 \%$ CI 0 to 0.19$)$.

A total number of nine adult samples were selected at random from $>170$ samples for comparison. The mean age of these adult subjects was 54.1 years (range 33-78). The presentations of the adults included were; chest pain $(n=1)$, gastrointestinal bleed $(n=3)$, back pain $(n=2)$, collapse $(n=1)$, head injury $(n=1)$ and breathlessness $(\mathrm{n}=1)$.

Figure 2 shows an example time profile of $\mathrm{m} / \mathrm{z} 59$ (acetone) in a preschool child (a) and its accompanying capnography trace (b) to highlight how the peaks on the time profile for acetone correspond with exhalation. This is compared with an adult sample (figure 3 ) and it is apparent that the profiles differ in that the adult sample shows smooth tidal breathing with a regular pattern emerging compared with the more irregular pattern in the profile of the preschool child. However, the comparable peaks of the mass spectrometric data with the capnography data illustrate that the mass spectrometer is analysing the exhaled breath of the preschool child in real time, providing instantaneous data.

\section{DTW distance of mass channels from $\mathrm{m} / \mathrm{z} 59$}

An example of DTW for m/z 59 and 15 is illustrated in figure 4 The average DTW distance of each mass channel
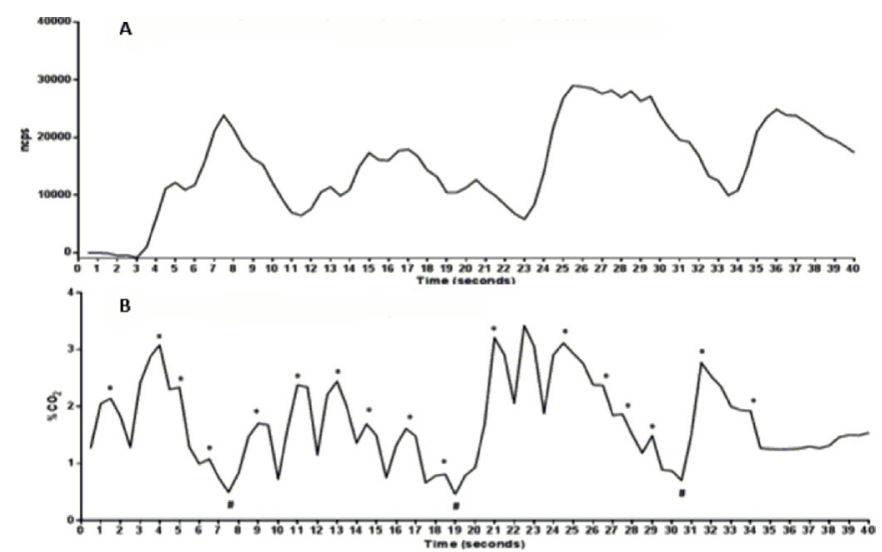

Figure 2 Example of channel 59 (acetone) for a preschool child $(\mathrm{A})$ and the accompanying capnography trace (B). from $\mathrm{m} / \mathrm{z} 59$ for all the child and adult samples were ranked from smallest to the largest, the top 20 are shown in Figure 5.

\section{DISCUSSION}

The principle aim of the study was to assess the feasibility and acceptability of collecting and analysing breath samples from preschool children using real-time PTRTOF-MS. We found that breath sampling in young children was well tolerated. Methods of distraction and maintaining a calm environment resulted in the successful

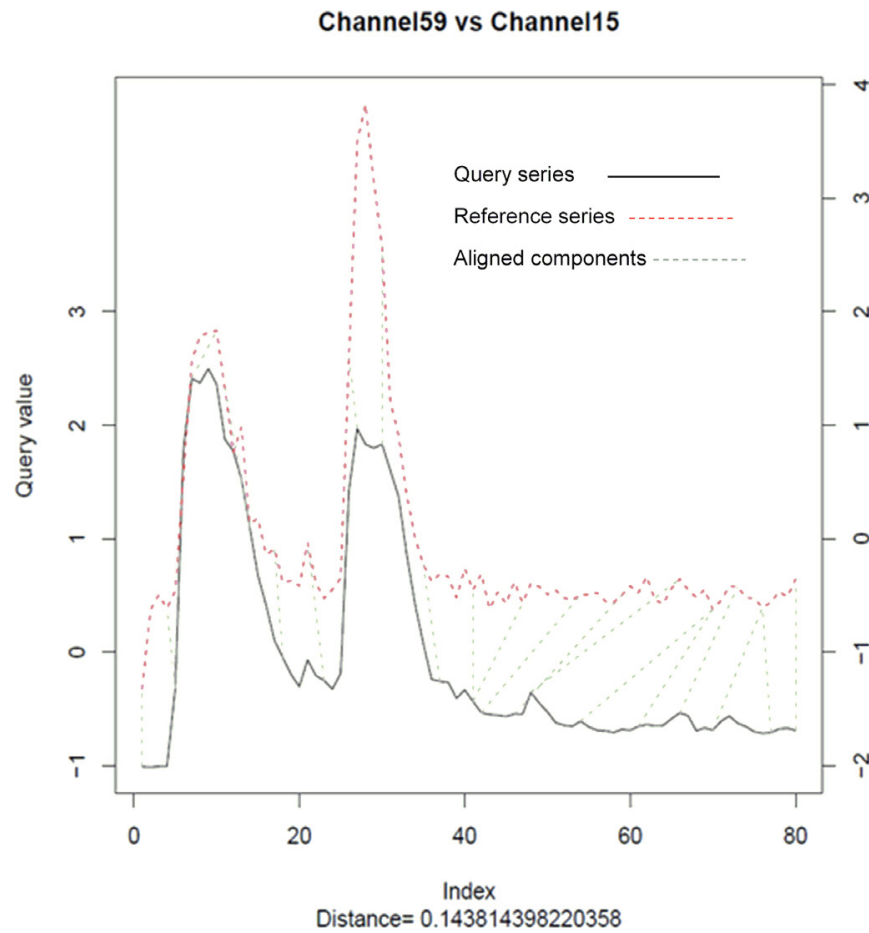

Figure 4 Example of dynamic time warping used to identify mass channels which have a real-time breath profile most similar to $\mathrm{m} / \mathrm{z} 59$ (acetone); comparison of the $\mathrm{m} / \mathrm{z} 15$ signal with the $\mathrm{m} / \mathrm{z} 59$ signal gives a DTW distance between series of 0.14 . The DTW distance is the sum of the distances between the aligned components of the reference series and a query series. DTW, dynamic time warping. 


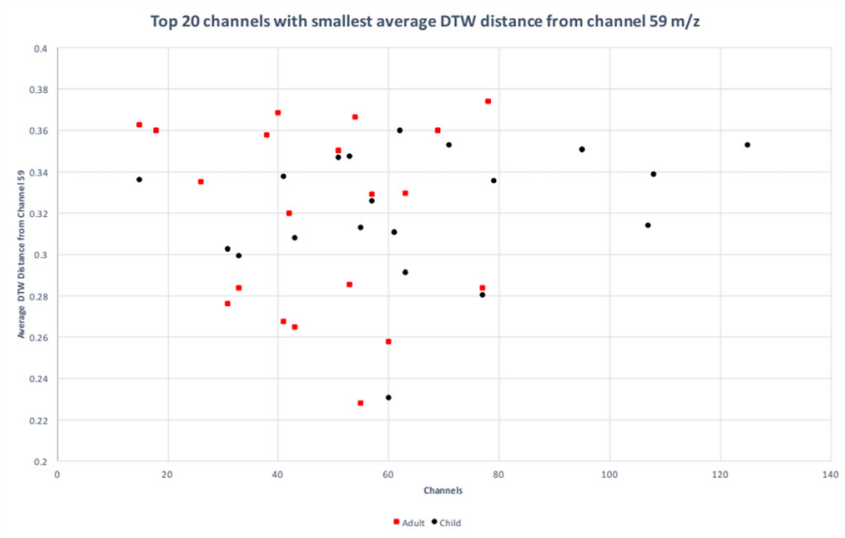

Figure 5 Top 20 channels with smallest average DTW distance from channel m/z 59 in preschool children (black square) and adults (red dot). DTW, dynamic time warping.

collection and analysis of analysable data from 11 of the 16 children included in the study. In the remaining five children, the samples were not analysable due to insufficient sample being collected. The primary reason for this was the children not keeping the mask on their face continuously for the required amount of time, resulting in leaks and failure to collect a fully analysable sample. This approach was clinically safe in children admitted with acute wheezing and minimal expiration was needed as tidal breathing reflects the child's normal breathing pattern.

Differences were observed in the sample profiles produced by paediatric and adult participants. The respiratory pattern is different in children and adults hence it is not surprising that they produce different VOC profiles. We show, however, that the relationship between the acetone and other channels is similar between adults and children. Despite acetone having a blood:air partition coefficient $>100$, that is, its high solubility allows for some exchange within the conductive airways, ${ }^{26}$ its abundance in breath provides a clear exhalation profile. Figure 1 demonstrates the use of acetone as a marker herein for assessing the breath profile. The end-tidal portion of the breath is often targeted as the desired portion of breath to analyse as it is believed to best represent VOCs from the alveolar region which best represent the metabolites endogenous to the body.

An important finding from this study is that we were able to collect samples of breath from preschool children and analyse them using online PTR-TOF-MS. The real-time similarity measurement from DTW provides a measure for comparing VOC profiles by correcting for differences in breathing pattern. Despite the differences in breathing patterns, of the top 10 VOC profiles based on the DTW scores, eight were common to both children and adults.

\section{Limitations}

We acknowledge that this is a single-centre pilot study in which children and adults were recruited from acute settings, mainly the emergency department. There is a potential for selection bias since the apparatus is operated by trained personnel, therefore patients could only be recruited at times when these staff were present. For those preschool children who were unable to provide an analysable sample, we did not perform interviews or administer questionnaires as part of this study. We acknowledge that this may have provided valuable feedback, however, this had not formed part of our research ethics committee application. Furthermore, there is unexplained variation in the PTR-TOF-MS profiles, and we do not know the relative contribution of disease factors to the pattern of VOCs we found because of the small pilot sample size. Equally, the contribution of patient factors such as respiratory rate or pattern, body mass and medication taken to VOC profiles was not attributable. In order to avoid experimental factors influencing the breath profiles as much as possible, the PTR-TOF-MS setup was kept constant for all children. The sampling lines, and breath device were kept small to reduce dead volume and the use of a ToF-MS detector ensured a fast and consistent acquisition rate. The use of DTW allowed assessment independent of breathing rate.

There are several key areas that need to be further explored in relation to this technology in young children. It is important that we understand why some groups of mass channels vary with the breath cycle (in both adults and children) and why others do not. Due to the more irregular breathing pattern in children, the end-expiratory phase of the breath cycle is harder to identify and an alternative may be to analyse throughout the entire breath cycle. Furthermore, the influences of different breathing patterns, environment, season, time of day, medication and disease phenotype need to be explored. This requires breath samples from large cohorts of children.

In summary, we investigated the feasibility of real-time breath sampling in a pilot study involving preschool children and found it an acceptable method for capturing breath profiles in the majority of patients. We found commonality of VOC profiles detected by online PTRTOF-MS in preschool children and adults. This combined with over two-thirds of preschool children providing an adequate sample demonstrates that this technology has potential as a non-invasive method to study metabolic processes associated with disease in preschool children.

Contributors KH, MM, PM, TC and EAG designed the study. MM and KH recruited the participants and collected data. MM, MW and MR analysed the data. KH, MM and MW prepared the manuscript with MW and MR creating the figures. The final draft was read and approved by all authors.

Funding The authors have not declared a specific grant for this research from any funding agency in the public, commercial or not-for-profit sectors.

Competing interests No, there are no competing interests.

Patient and public involvement Patients and/or the public were not involved in the design, or conduct, or reporting, or dissemination plans of this research.

Patient consent for publication Not required. 
Ethics approval This study received ethical approval from the National Research Ethics Committee East Midlands (UK), study number 09/H0403/92. All parents gave written informed consent.

Provenance and peer review Not commissioned; externally peer reviewed.

Data availability statement Data are available upon reasonable request. Data is available upon reasonable request.

Open access This is an open access article distributed in accordance with the Creative Commons Attribution Non Commercial (CC BY-NC 4.0) license, which permits others to distribute, remix, adapt, build upon this work non-commercially, and license their derivative works on different terms, provided the original work is properly cited, appropriate credit is given, any changes made indicated, and the use is non-commercial. See: http://creativecommons.org/licenses/by-nc/4.0/.

ORCID iD

Karl Holden http://orcid.org/0000-0002-1420-8959

\section{REFERENCES}

1 Martinez FD, Wright AL, Taussig LM, et al. Asthma and wheezing in the first six years of life. N Engl J Med Overseas Ed 1995;332:133-8.

2 Gaillard EA, McNamara PS, Murray CS, et al. Blood eosinophils as a marker of likely corticosteroid response in children with preschool wheeze: time for an eosinophil guided clinical trial? Clin Exp Allergy 2015;45:1384-95.

3 Castro-Rodriguez JA, Rodrigo GJ. Efficacy of inhaled corticosteroids in infants and preschoolers with recurrent wheezing and asthma: a systematic review with meta-analysis. Pediatrics 2009;123:e519-25.

4 Poli D, Carbognani P, Corradi M, et al. Exhaled volatile organic compounds in patients with non-small cell lung cancer: cross sectional and nested short-term follow-up study. Respir Res 2005;6:71.

5 Barker M, Hengst M, Schmid J, et al. Volatile organic compounds in the exhaled breath of young patients with cystic fibrosis. Eur Respir $J$ 2006;27:929-36.

6 Neerincx AH, Vijverberg SJH, Bos LDJ, et al. Breathomics from exhaled volatile organic compounds in pediatric asthma. Pediatr Pulmonol 2017;52:1616-27.

7 Barnes PJ. Reactive oxygen species and airway inflammation. Free Radic Biol Med 1990;9:235-43.

8 Phillips M, Herrera J, Krishnan S, et al. Variation in volatile organic compounds in the breath of normal humans. $J$ Chromatogr $B$ Biomed Sci Appl 1999;729:75-88.

9 Wilde MJ, Cordell RL, Salman D, et al. Breath analysis by twodimensional gas chromatography with dual flame ionisation and mass spectrometric detection - method optimisation and integration within a large-scale clinical study. J Chromatogr A 2019;1594:160-72.

10 O'Hara ME, O'Hehir S, Green S, et al. Development of a protocol to measure volatile organic compounds in human breath: a comparison of rebreathing and on-line single exhalations using proton transfer reaction mass spectrometry. Physiol Meas 2008;29:309-30.

11 Pleil JD, Lindstrom AB. Measurement of volatile organic compounds in exhaled breath as collected in evacuated electropolished canisters. J Chromatogr B Biomed App/ 1995;665:271-9.

12 Blake RS, Monks PS, Ellis AM. Proton-transfer reaction mass spectrometry. Chem Rev 2009;109:861-96.

13 Smith D, Španěl P, Direct paněl P. Direct, rapid quantitative analyses of BVOCs using SIFT-MS and PTR-MS obviating sample collection. TrAC Trends in Analytical Chemistry 2011;30:945-59.

14 Dallinga JW, Robroeks CMHHT, van Berkel JJBN, et al. Volatile organic compounds in exhaled breath as a diagnostic tool for asthma in children. Clin Exp Allergy 2010;40:68-76.

15 van de Kant KDG, van Berkel JJBN, Jöbsis Q, et al. Exhaled breath profiling in diagnosing wheezy preschool children. Eur Respir $J$ 2013;41:183-8

16 van Mastrigt E, de Jongste JC, Pijnenburg MW. The analysis of volatile organic compounds in exhaled breath and biomarkers in exhaled breath condensate in children - clinical tools or scientific toys? Clin Exp Allergy 2015;45:1170-88.

17 van der Schee MP, Hashimoto S, Schuurman AC, et al. Altered exhaled biomarker profiles in children during and after rhinovirusinduced wheeze. Eur Respir J 2015;45:440-8.

18 Trefz P, Obermeier J, Lehbrink R, et al. Exhaled volatile substances in children suffering from type 1 diabetes mellitus: results from a crosssectional study. Sci Rep 2019;9:1-9.

19 Obermeier J, Trefz P, Happ J, et al. Exhaled volatile substances mirror clinical conditions in pediatric chronic kidney disease. PLoS One 2017:12:e0178745.

20 Blake RS, Whyte C, Hughes CO, et al. Demonstration of protontransfer reaction time-of-flight mass spectrometry for realtime analysis of trace volatile organic compounds. Anal Chem 2004;76:3841-5.

21 Wyche KP, Blake RS, Ellis AM, et al. Technical note: performance of chemical ionization reaction time-of-flight mass spectrometry (CIR-TOF-MS) for the measurement of atmospherically significant oxygenated volatile organic compounds. Atmos Chem Phys 2007;7:609-20

22 Spanel P, Dryahina K, Smith D. The concentration distributions of some metabolites in the exhaled breath of young adults. $J$ Breath Res 2007;1:026001.

23 Spaněl P, Dryahina K, Smith D. Acetone, ammonia and hydrogen cyanide in exhaled breath of several volunteers aged 4-83 years. $J$ Breath Res 2007;1:011001.

24 Giorgino T. Computing and visualizing dynamic time warping alignments in R : the dtw package. J Stat Softw 2009;31:1-24.

25 Garcia-Marcos L, Edwards J, Kennington E, et al. Priorities for future research into asthma diagnostic tools: a PAN-EU consensus exercise from the European asthma research innovation partnership (EARIP). Clin Exp Allergy 2018;48:104-20.

26 King J, Unterkofler K, Teschl G, et al. A mathematical model for breath gas analysis of volatile organic compounds with special emphasis on acetone. J Math Biol 2011;63:959-99. 